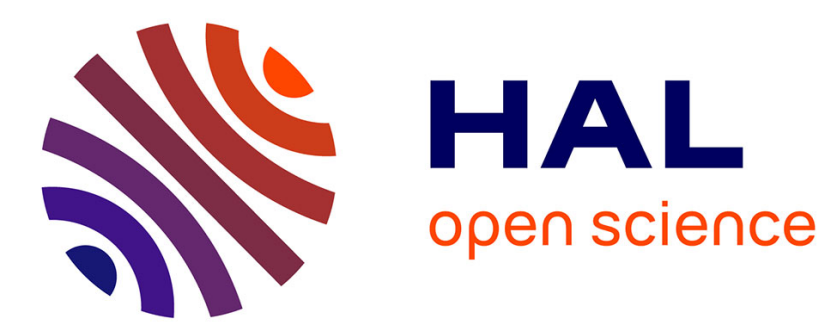

\title{
Continuous Optimal Control Approaches to Microgrid Energy Management
}

Benjamin Heymann, J. Frederic Bonnans, Pierre Martinon, Francisco Silva, Fernando Lanas, Guillermo Jimenez

\section{- To cite this version:}

Benjamin Heymann, J. Frederic Bonnans, Pierre Martinon, Francisco Silva, Fernando Lanas, et al.. Continuous Optimal Control Approaches to Microgrid Energy Management. Energy Systems, 2018, 9 (1), pp.59-77. hal-01129393v2

\section{HAL Id: hal-01129393 \\ https://inria.hal.science/hal-01129393v2}

Submitted on 13 Mar 2015

HAL is a multi-disciplinary open access archive for the deposit and dissemination of scientific research documents, whether they are published or not. The documents may come from teaching and research institutions in France or abroad, or from public or private research centers.
L'archive ouverte pluridisciplinaire HAL, est destinée au dépôt et à la diffusion de documents scientifiques de niveau recherche, publiés ou non, émanant des établissements d'enseignement et de recherche français ou étrangers, des laboratoires publics ou privés. 


\title{
Continuous Optimal Control Approaches to Microgrid Energy Management
}

\author{
Benjamin Heymann, J. Frédéric Bonnans, Pierre Martinon, Francisco Silva, Fernando Lanas and \\ Guillermo Jimenez Senior Member, IEEE
}

\begin{abstract}
We propose a novel method for the microgrid energy management problem by introducing a continuous-time, rolling horizon formulation. The energy management problem is formulated as a deterministic optimal control problem (OCP). We solve (OCP) with two classical approaches: the direct method [1], and Bellman's Dynamic Programming Principle (DPP) [2]. In both cases we use the optimal control toolbox BOCOP [3] for the numerical simulations. For the DPP approach we implement a semi-Lagrangian scheme [4] adapted to handle the optimization of switching times for the on/off modes of the diesel generator. The DPP approach allows for an accurate modeling and is computationally cheap. It finds the global optimum in less than 3 seconds, a CPU time similar to the Mixed Integer Linear Programming (MILP) approach used in [5]. We achieve this performance by introducing a trick based on the Pontryagin Maximum Principle (PMP). The trick increases the computation speed by several orders and also improves the precision of the solution. For validation purposes, simulation are performed using datasets from an actual isolated microgrid located in northern Chile. Results show that DPP method is very well suited for this type of problem when compared with the MILP approach.
\end{abstract}

Index Terms-Direct method, electricity production, Energy Management System (EMS), Dynamic Programming Principle (DPP), Mixed Integer Linear Programming (MILP), optimal control, Pontryagin Maximum Principle (PMP), semi-Lagrangian scheme, smart grid.

\section{INTRODUCTION}

A MICROGRID is a group of interconnected loads and distributed energy resources that acts as a single controllable entity. It can operate connected to the main network or autonomously (isolated). In either case, an Energy Management System (EMS) is required to coordinate the different units that composes it. As described in [6], the microgrid optimal energy management problem falls into the category of mixed integer nonlinear programming. Thus, the objective function and constraints may include functions of second or higher order polynomial equations with some start-up/shutdown variables. Complex constraints are involved to model the operational limitations of generation / storage devices or to represent controllable loads and commitment decisions. Furthermore, considering network constraints (load flow) adds another degree of complexity to the microgrid optimal energy management problem. To handle and solve these problem formulations, heuristic optimization techniques have been applied, including Genetic Algorithms (GA) [7], [8], PSO [7], and Ant Colony Optimization (ACO) [9]; the problem formulation has also been relaxed by incorporating the inequality constraints in the objective function using penalty factors. The minimization of total operating cost in stand-alone operation, and maximization of microgrids revenue in grid connected mode are two typically pursued objectives in secondary control. Nevertheless, MILP formulations have been also developed presenting adequate results but with difficulties for modeling battery charging/discharging behavior, and the diesel engine costs [6]. However, some approaches have also incorporated the reduction of Greenhouse gas (GHG) emissions as an additional objective for the microgrid operation. In this case, the energy management problem is formulated as a multi-objective optimization problem and solved with different techniques [6]. Pareto optimal solutions are investigated in [9] and [10] by using PSO and ACO techniques, respectively, while a weighted objective function that combines different individual objective functions, together with heuristic optimization techniques, are used in [11], [12]. Finally, recent works, focusing on micro grid energy management systems, have incorporated a more detailed modeling of the energy storage system, considering the importance of the cost associated with its replacement, so that extending the life span of the battery is part of the objective. In this context, GA have been implemented to solve the problem [13], and other predictive control approaches such as the ones described in [14], [15], [16], [17].

Considering the challenges involved with the microgrid EMS problem such as units modeling, CPU solving time for real applications and switching among others, a microgrid model formulation as a continuous time, optimal control problem is presented in this work. This approach keeps the original non-linear model for the numerical optimization, which may give more accurate solutions. The proposal considers two solution methods that are detailed as follows: The direct method uses a time discretization to transform the continuous optimal control problem into a Nonlinear Programming (NLP) problem. The (NLP) is then solved with any usual technique, see for instance [1]. The DPP method is based on Bellmans Principle and uses a discretization of both the time and space to compute the value function. This information then allows to reconstruct the optimal trajectory, see for example [2].

Numerical simulations are performed for both methods using the optimal toolbox BOCOP [3]. The proposed methods are validated based on a real microgrid operating in Huatacondo, an isolated northern Chilean village that relies completely on the microgrid concept for its electricity supply, which is described in section II. The present study uses a similar model to the one presented in [5], so that the comparison is relevant showing obtained results for the three approaches, MILP, direct method and DPP.

The paper is organized as follows. Section II describes 
the microgrid system and the optimal control formulation for its energy management. Section III explains the numerical methods we use to solve the optimal control problem. Section IV presents the numerical simulations with the direct and DPP methods. Section V comments the results of the simulations. The conclusion sums up the main results and presents ongoing research in the continuation of this work.

March 11, 2015

\section{Model Presentation}

\section{A. General Aspects}

1) Description of the Microgrid: The following model is based on a real microgrid operating in Huatacondo, an isolated northern Chilean village that relies completely on the microgrid concept for its electricity supply. The microgrid consists of a photovoltaic (PV) power plant, a diesel generator and a Battery Energy Storage System (BESS). It uses a mix of fuel and renewable energy sources. The solar panel and the wind turbine produce electricity without any additional cost, but the generation pattern cannot be controlled, and depends on the everyday weather. The BESS can store energy for later use, but has a limited capacity and power. The diesel generator has a minimal and maximal output level, and has a fixed start-up cost. All these are local generation units, i.e. situated physically near the electrical consumption, and we do not consider electrical losses due to distribution. The aim is to find the optimal planning that meets the power demand and minimizes the operational costs, which in this case mainly relates to the diesel consumption. We follow the problem description from [5].

2) Optimal Control Formulation: We take a fixed horizon $T=48$ hours. For $t \in[0, T]$, we denote by $P_{S}(t)$ the solar power from the photovoltaic panels, $P_{D}(t)$ the diesel generator power and $P_{L}(t)$ the electricity load. The state of charge $S O C(t)$ of the BESS evolves according to the dynamics

$$
S \dot{O} C(t)=\frac{1}{Q_{B}}\left(P_{I}(t) \rho_{I}-P_{O}(t) / \rho_{O}\right),
$$

where $Q_{B}$ is the maximum capacity of the battery, $P_{I}, P_{O}>0$ are the input and output power of the BESS, and $\rho_{I}, \rho_{o} \in[0,1]$ are the efficiency ratios for the charge and discharge processes, assumed constant.

We also introduce the slack variable $P_{\text {slack }}$ that represents the excess power $\left(P_{\text {slack }}<0\right)$, which have to be shedded, or missing power in the microgrid $\left(P_{\text {slack }}>0\right)$, which turns into unserved energy for the consumers. From the mathematical point of view, this ensures feasibility of the problem. From the practical point of view, a cost is associated to the unserved power, associated with a fine.

The underlying power equilibrium equation is

$$
P_{D}+P_{O}+P_{S}+P_{\text {slack }}-P_{L}-P_{I}=0
$$

Taking into account the demand and the various power production devices, we obtain that $P_{O}$ and $P_{I}$ can be written as nonlinear functions of $\left(t, P_{D}\right)$ :

$$
\begin{aligned}
& P_{O}\left(t, P_{D}\right)=-\min \left(0, P_{S}+P_{D}-P_{L}+P_{\text {slack }}\right) \\
& P_{I}\left(t, P_{D}\right)=\max \left(0, P_{S}+P_{D}-P_{L}+P_{\text {slack }}\right) .
\end{aligned}
$$

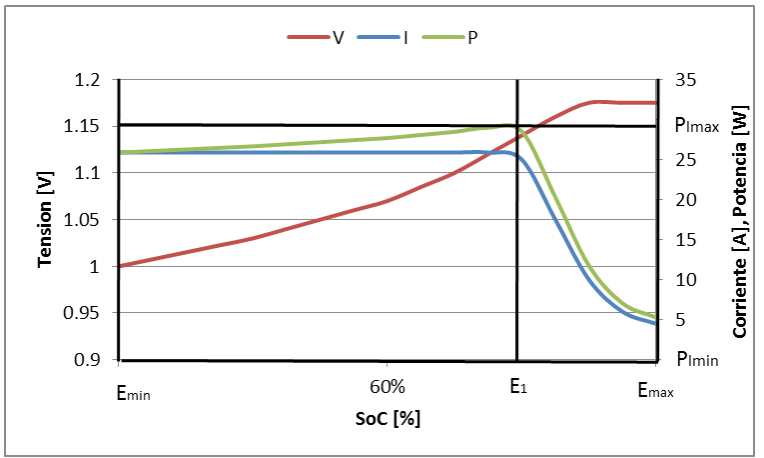

Fig. 1: Battery charge constraint

We model the fuel consumption of the diesel generator by the following strictly concave function

$$
\int_{0}^{T} K P_{D}(t)^{0.9} d t
$$

with $K=0.471426$ obtained by regression on data from the actual generator consumption.

For physical reasons, the system is subject to the following constraints at every time $t \in[0, T]$ :

$$
\begin{gathered}
S O C(t) \in[0.2,1] \\
P_{D}(t) \in\{0\} \cup\left[P_{\text {min }}, P_{\text {max }}\right] \\
\left\{\begin{array}{l}
P_{I}\left(P_{D}(t), t\right) \in[0,13.2] \quad \text { if } S O C(t)<0.9 \\
P_{I}\left(P_{D}(t), t\right) \leq 1320(S O C(t)-1)^{2} \quad \text { otherwise }
\end{array}\right. \\
P_{O}(t) \in[0,40],
\end{gathered}
$$

Note that (3) implies that (7)-(8) are constraints on $P_{D}$. The state constraint (5) expresses the maximum and minimum charge of the battery. Constraints (6) to (8) are control constraints. The minimal and maximal power for the diesel generator are given by (6). The charging and discharging limits for the battery are stated in (7) and (8). The charging limit depends on the state of charge, and is therefore a mixed control-state constraint, as illustrated on Fig. 1.

Since the operations time frame is larger than the optimization horizon, we impose a constraint on the final time to avoid the battery depletion. This constraint will be imposed through either a periodicity condition $S O C(0)=S O C(T)$ (direct method) or a penalization term $g(S O C(T))$ (DPP method).

To sum up, the optimal control problem to be solved can be written under the following abstract formulation

$$
(O C P)\left\{\begin{aligned}
& \min _{u} \int_{0}^{T} \ell(u(t)) d t+g(x(T)) \\
& \dot{x}(t)=F(u(t), t) \\
& x(0)=x_{0} \\
& u(t) \in U_{x(t)} \\
& x(t) \in \mathcal{C} .
\end{aligned}\right.
$$

In the notation above, $x$ is the state variable $(S O C), u$ is the control variable $\left(P_{D}\right), F$ is the dynamics of the system, 
$U_{x(t)}$ corresponds to the control constraints on $u(t)$ that may depend on the value of $x(t)$, and $\mathcal{C}$ corresponds to the state constraints.

\section{B. Switching cost}

Turning the diesel generator on consumes fuel. We model this by considering that the diesel generator has two modes: when off, the only admissible control is $P_{D}=0$, whereas when it is on, $P_{D} \in\left[P_{\min }, P_{\max }\right]$. At any time, one can switch from one mode to the other by paying the associated cost. It should be stressed out that while the modeling of the switching cost is made straightforward by the Dynamic Programming approach, it is challenging for the Direct Method approach.

\section{PRESENTATION OF THE NUMERICAL METHODS}

We give here a brief presentation of the two resolution approaches we are considering and explain how to apply them for solving (9). The interested reader will find more on those approaches in [4], [18].

\section{A. The Direct Method Approach}

1) Presentation: In the Direct Method we apply a time discretization to the dynamics equation. The optimal control problem is rewritten as a finite-dimensional optimization problem. The "decision" variables of this discretized problem are the values of the control variables for each time steps. Since the discretized problem is solved by locally convergent algorithms, we cannot guarantee that the obtained solution (if any) is close to a global optimum. On the other hand, this approach often provides efficient solutions for large optimal control problems, with limited computing times.

Summary of the time discretization, using the Euler formula:

$$
\begin{array}{ll}
t \in[0, T] & \rightarrow\left\{t_{0}=0, \ldots, t_{N}=T\right\} \\
x(\cdot), u(\cdot) & \rightarrow Z=\left\{x_{0}, \ldots, x_{N}, u_{0}, \ldots, u_{N-1}\right\} \\
\hline \text { Criterion } \rightarrow \min h \sum_{i=0}^{N-1} \ell\left(u_{i}\right)+G\left(x_{N}\right) \\
\text { Dynamics } \rightarrow x_{i+i}=x_{i}+h f\left(x_{i}, u_{i}\right) \quad i=0, \ldots, N \\
\text { Controls } \rightarrow u_{i} \in U_{x_{i}} \quad i=0, \ldots, N-1 \\
\text { States } \rightarrow x_{i} \in \mathcal{C} \quad i=0, \ldots, N
\end{array}
$$

We therefore obtain a nonlinear programming problem on the discretized state and control variables

$$
(N L P)\left\{\begin{array}{l}
\min _{Z} F(Z) \\
L B \leq C(Z) \leq U B
\end{array}\right.
$$

In the optimal control toolbox BocOP the discretized nonlinear optimization problem is solved by the IPOPT solver [19] that implements a primal-dual interior point algorithm.

2) Modeling Remarks: This method allows a periodicity constraint of the form $S O C(0)=S O C(T)$ where the actual value is optimized by the algorithm. On the other hand, the constraint (6) is simplified into $P_{D} \in\left[0, P_{\max }\right]$. Another drawback is that switching costs are binary decisions which are not easily handled in this framework. Finally, the cost function is regularized by a small quadratic term $\epsilon \int_{0}^{T} P_{O}^{2}(t) \mathrm{d} t$ in order to improve convergence.

\section{B. Dynamic Programming Approach}

In order to solve the DPP, we propose a semi-Lagrangian scheme, which is adapted for problems with switching modes. We refer the reader to [4] and the references therein for an introduction to semi-Lagrangian schemes applied to optimal control problems. In addition, the Pontryagin Maximum Principle (PMP), see [20], can be used to give additional information on the optimal solution, which allows to significantly reduce the computational effort of the method.

1) Brief Presentation of the Theory: Let $V\left(t, x_{0}\right)$ denote the value of problem (9) with initial time $t$ and initial condition $x_{0}$. In R. Bellman's words [2] "An optimal policy has the property that whatever the initial state and initial decision are, the remaining decisions must constitute an optimal policy with regard to the state resulting from the first decision." In mathematical terms, $V$ satisfies for $h \in(0, T-t)$ :

$$
V\left(t, x_{0}\right)=\inf \left\{\int_{t}^{t+h} \ell\left(u_{t}\right) \mathrm{d} s+V(t+h, x(t+h))\right\},
$$

the infimum being taken over the set of admissible controls. In our case, we will use an extended version of the DPP approach that handles the switchings.

2) Semi-Lagrangian scheme: The Semi-Lagrangian scheme consists in solving backward in time a discretization of (10) over the space. We have chosen this scheme to solve the problem because it has good stability properties, it allows large time steps and it is easy to implement. Let us motivate the scheme by first discretizing in time (10). Given a time step $h$ and $N$ such that $N h=T$, let us set $t_{k}=k h(k=0, \ldots, N)$. Denoting by $V^{k}$ the "approximated" value function at $t_{k}$ we have

$$
V^{k}(x)=\min _{u \in U_{x}}\left\{h \ell(u)+V^{k+1}\left(x+h F\left(u, t_{k}\right)\right)\right\} .
$$

The Semi-Lagrangian scheme is obtained from (11), by discretizing in space the state variable $x$ and introducing interpolation operators in order to approximate $V^{k+1}\left(x+h F\left(u, t_{k}\right)\right)$ in terms of its values in the space grid. The scheme is solved backward in time and, under standard conditions, it is shown that it converges to the solution $V$ of (10).

3) The PMP trick: The problem has an interesting property that greatly reduces the numerical computations. If $\bar{u}$ is the optimal control, denote by $\bar{x}$ the optimal state and by $\bar{p}$ the Lagrange multiplier associated to the dynamics constraint $\dot{x}(t)=F(t, u(t))$. Defining the Hamiltonian $H(u, p, t):=$ $p F(u, t)+\ell(u)$ the PMP says that for all $t \in[0, T]$ we have

$$
H(\bar{u}(t), \bar{p}(t), t) \leq H(v, \bar{p}(t), t) \text { for all } v \in U_{\bar{x}(t)} .
$$

Since $v \mapsto H(v, \bar{p}(t), t)$ is piecewise strictly concave, it can attain its minimum only at one of the extreme points of the pieces. Taking also into account the constraints, we have at most five possible optimal controls, as illustrated in figure 2. Moreover, the values of those controls can be computed explicitly, since they do not depend on $\bar{p}$. Therefore, when doing the minimization in (11), we can test only those controls instead of discretizing the control space, gaining both in speed and precision. 


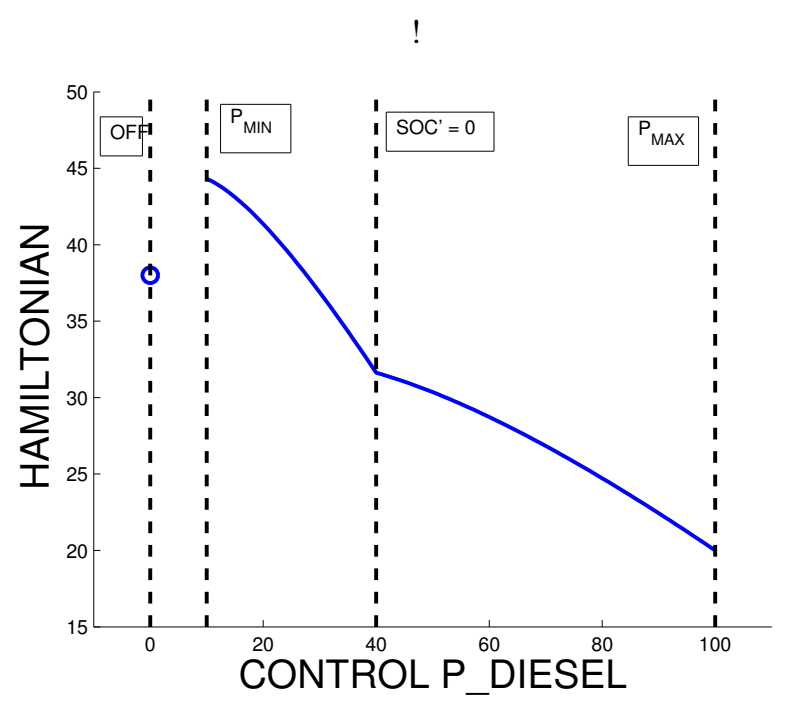

Fig. 2: The PMP trick illustrated

- if the Diesel is off (mode 0 ), we simply take $P_{D}=0$.

- if the Diesel is on (mode 1), we test the five cases

- $P_{D}=P_{\min }$ (minimum power),

- $P_{D}=P_{\max }$ (maximum power),

- $P_{D}$ such that $S \dot{O} C=0$ (battery unused),

- $P_{D}$ such that $P_{i}=P_{i}^{\max }(S O C)$ (maximal charge),

- $P_{D}$ such that $P_{0}=40$ (maximal discharge).

It should be noted that the specific structure of the problem permits such a computational simplification. More precisely, we use the fact that all the candidates values for the optimal control do not depend on the adjoint state $p$ and therefore can be evaluated and tested when computing the value function. In the general case, the control that minimizes the Hamiltonian is expressed from both the state and adjoint state, the latter being unavailable in the DPP approach (the adjoint actually corresponds to the gradient of the Value Function).

Remark 3.1: Slack variable In the five cases above, when we set the diesel power, we recompute the battery power from the power equilibrium (2), then adjust the slack variable if needed. When we set the battery power, we then recompute the diesel power from (2) and adjust the slack likewise.

4) Periodicity condition: We model the periodicity condition by taking a similar approach to the "big M method" in linear programming:

$$
\begin{array}{ll}
g(\operatorname{SOC}(T))=M & \text { if } S O C(T)<S O C_{0} \\
g(\operatorname{SOC}(T))=0 & \text { if } \operatorname{SOC}(T) \geq S O C_{0}
\end{array}
$$

For the following simulations, we set $S O C_{0}=0.7$.

\section{NUMERICAL SIMULATIONS}

\section{A. Comments on the inputs: solar power and power load}

We test the algorithms on two historical data sets. Both data sets correspond to the two most representative 24 hours days, one data set was obtained with winter data, the other one with summer data. Figure 3 and 4 show the load power and the solar power for the two days of each period.

Given the situation of the actual microgrid in the Atacama desert, we assume the production from the photovoltaic panels

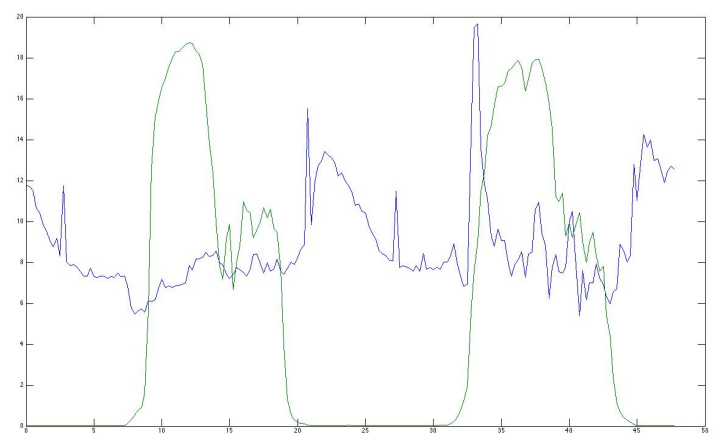

Fig. 3: Summer data, the bell shaped curve is the solar production, and the other one is the demand

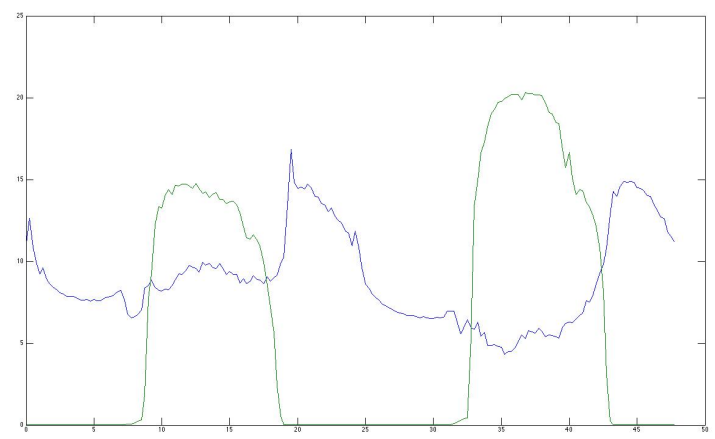

Fig. 4: Winter data, the bell shaped curve is the solar production, and the other one is the demand

to be reliably predictable, with small variance and almost determistic. The demand on the other hand has a greater variability. While in this initial work it is modeled as deterministic, its stochastic modeling is under study.

\section{B. Optimal solutions for the different methods}

In addition to the direct and DPP methods, we also present the results obtained with the MILP approach from [5] as baseline for comparison. The 6 solutions are illustrated in Figure 5 and 6 for the DPP approach, in Figure 7 and 8 for the direct approach and in Figure 9 and 10 for MILP method. The numerical results are summarized in Table I.

$\begin{array}{llll} & \text { MILP } & \text { DIRECT } & \text { DPP } \\ \text { Diesel range } & {[18.66,29.69]} & {[2.63,14.23]} & {[8.15,28.66]} \\ \text { Switchings } & 2 & 3 & 2 \\ \text { Total Cost } & 34785 & 36244 & 34378 \\ \text { Cpu Time } & 3.92 \mathrm{~s} & 4.41 \mathrm{~s} & 0.88 \mathrm{~s} \\ \text { SOC }(0)=\text { SOC(T) } & 0.7 & 0.641 & 0.7 \\ \text { SOC range } & {[0.20,0.89]} & {[0.38,0.75]} & {[0.30,0.83]} \\ \text { Slack Range } & {[0,0]} & {[0,0]} & {[0,0]}\end{array}$

TABLE I: Results: MILP, direct and DPP (summer case)

Discussion on the solutions:

- MILP and DPP solutions are quite close, while direct solution shows some clear differences (different initial/final SOC, no minimal power, spurious switchings) 


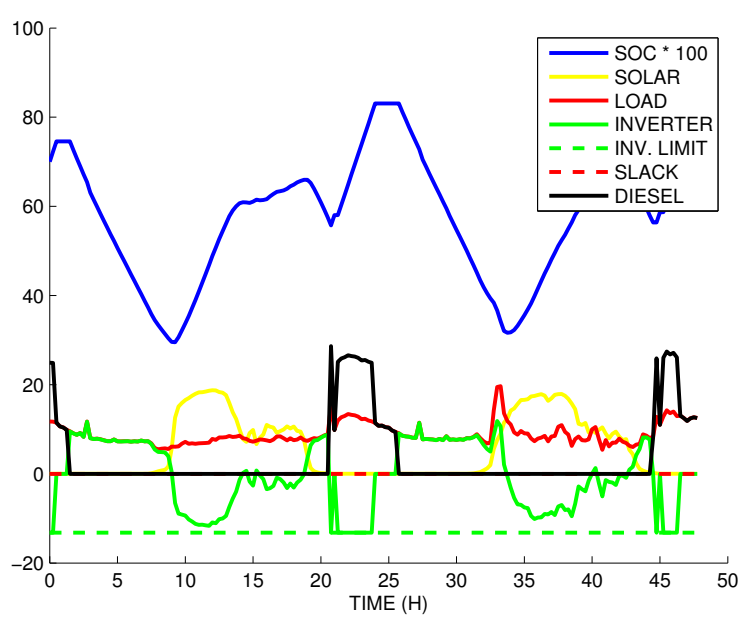

Fig. 5: Summer DPP Simulation

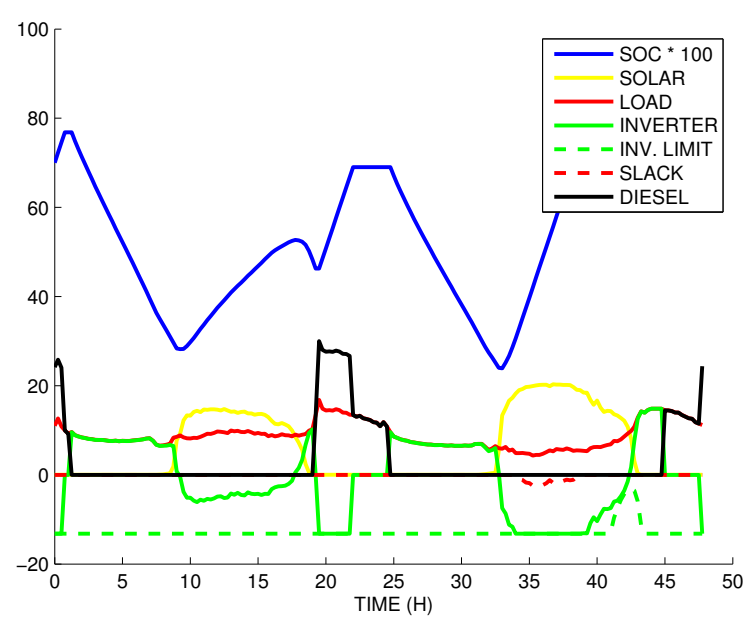

Fig. 6: Winter DPP Simulation

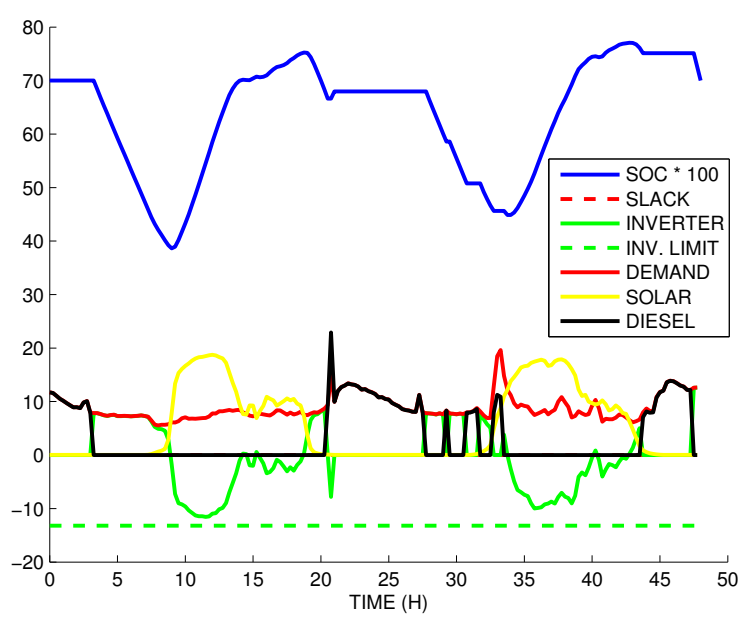

Fig. 7: Summer Direct Simulation

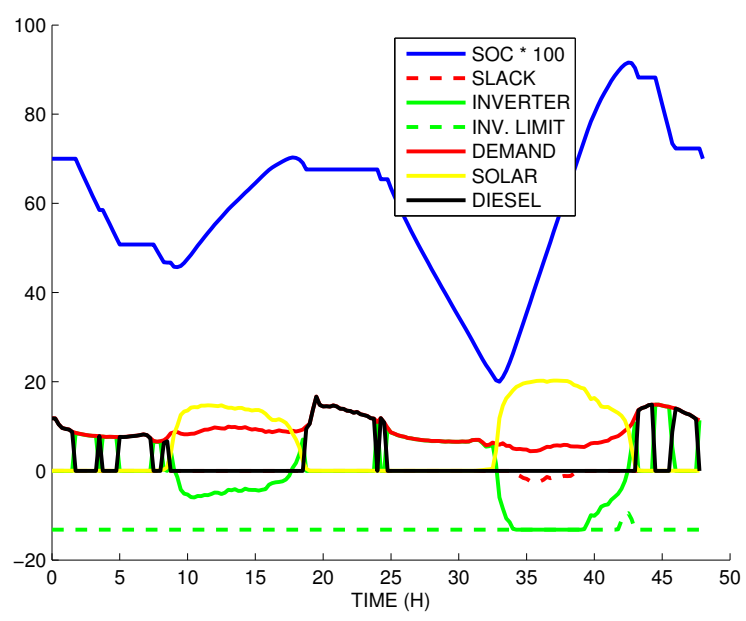

Fig. 8: Winter Direct Simulation

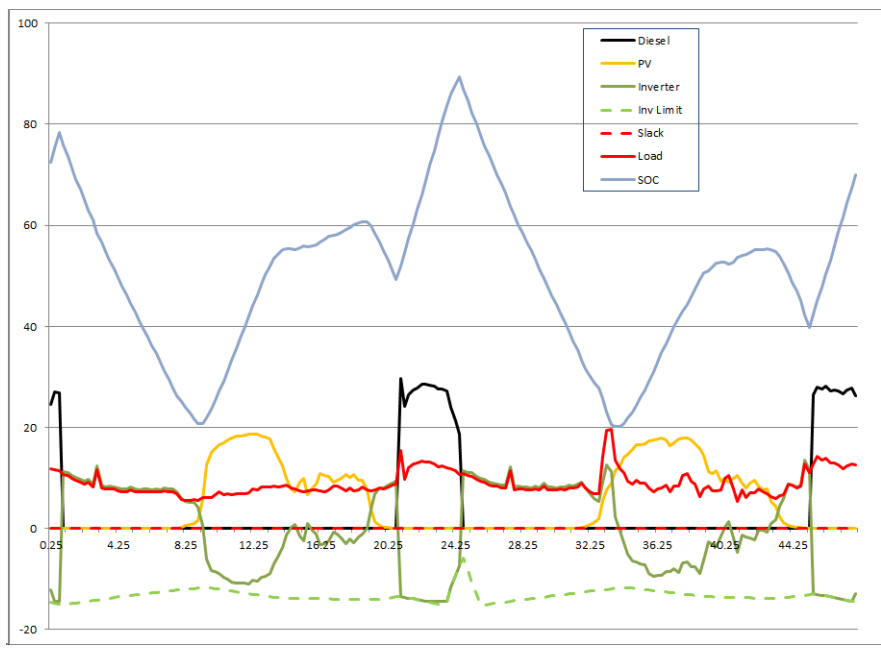

Fig. 9: Summer MILP Simulation

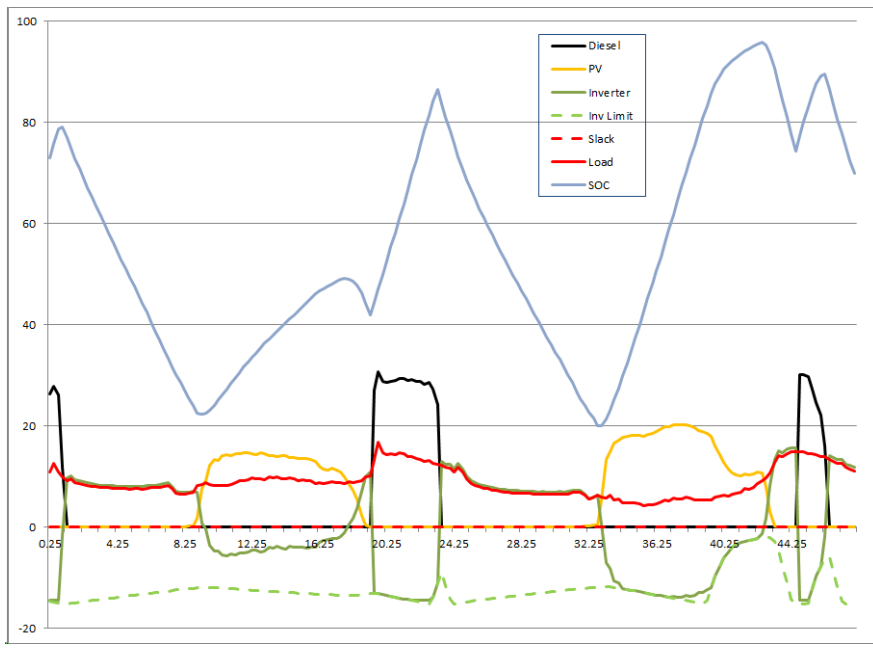

Fig. 10: Winter MILP Simulation power used to charge the battery

- Diesel is always off when solar power is available, and 
is switched on once a day during the evening peak in demand. Diesel output is often greater than power demand. At this times it is used also to charge the battery

- The battery fills the gaps between solar and diesel power in order to meet the demand, especially at night

- A qualitative difference between MILP and DPP/direct is the existence of time intervals with a constant SOC, while the diesel exactly matches the power load. In the MILP solutions the diesel is either off or saturating the maximal charge limit. This behaviour seems consistent with the concavity of the diesel comsumption with respect to power output (i.e. the efficiency of the diesel is better at higher output). On the other hand, both the direct and DPP solutions present intervals with constant SOC, and the PMP confirms this situation to be a possible optimum. This point would deserve closer investigation, for instance is this discrepancy due to the linearization performed in MILP ?

\section{Comparison of the methods}

We highlight below the differences between the three different optimization methods.

Global optimum : Both the MILP and direct approaches are local methods and may converge to a local solution, depending on the provided starting point and the choice of the stopping criterion (gap). On the other hand, the DPP approach performs a global optimization over all possible (discretized) trajectories, and therefore always finds the global optimum. This is an advantage for the user since one does not have to find a "suitable" starting point. Also, the DPP solution has a control in feedback form, whereas MILP and direct solutions are open-loop.

Switching cost : Both MILP and DPP approaches take into account the switching costs for the diesel generator. They typically find solutions with one switch per day, located during the peak of power demand in the evening. On the other hand, the direct approach has free switchings, which explains why it may find solutions with lots of on-off oscillations. This bad behaviour, however, can be partially alleviated by using a regularization of the cost function.

Nonlinear model : The MILP method requires a piecewise linear reformulation of the nonlinear functions in the model, here for example the charging power limit or diesel consumption. Both direct and DPP methods use the original nonlinear model. This simplifies the actual implementation, and may also give more accurate solutions.

Periodicity constraint and minimal diesel power : Compared to MILP and DPP, the direct method is able to optimize the value of the initial/final SOC, instead of fixing it. On the other hand, it does not take into account the minimal power output for the diesel generator.

Computation time : For this problem the computation time is a few seconds for MILP and direct method, and less than one second for the DPP approach. An interesting question is how well each method would scale for higher dimensions. MILP and direct approach are iterative methods, so changing the problem size may also lead to a different convergence, making it difficult to asses the evolution of the CPU time. For the DPP approach, on the other hand, the number of operations is always known and the CPU time can be predicted reliably. Due to the state discretization, however, adding new state variables to the problem would have a significant impact on performance (the so-called curse of dimensionality). In terms of high performance computing, parallelization is possible with MILP and DPP methods, not so easily with the direct method.

\section{CONCLUSION AND PERSPECTIVES}

We have applied two methods from the continuous optimal control field to the optimal energy management of a microgrid, namely the direct and DPP approaches. Numerical simulations indicate that the DPP methods is very well suited to this problem. We were able to obtain the global optimum in less than one second of CPU time, while taking into account the switching cost for the diesel generator. Solutions are close to the ones obtained in [5] with a MILP formulation, the main difference being the existence of time intervals where the battery stays at a constant SOC.

Ongoing work on this topic include a deeper investigation of the optimality conditions from Pontryagin's Principle, the extension to a stochastic model for the power demand, and the study of the long-term aging of the battery.

APPENDIX

MODEL PARAMETERS

\begin{tabular}{|l|l|l|l|}
\hline Name & Notation & Value & Unit \\
\hline Min Diesel Power & $P_{\min }$ & 5 & $\mathrm{~kW}$ \\
\hline Max Diesel Power & $P_{\max }$ & 120 & $\mathrm{~kW}$ \\
\hline Max ESS Power & $Q_{b a t}$ & 117 & $\mathrm{kWh}$ \\
\hline Unserved Energy Cost & $C_{U S}$ & 250 & $\mathrm{CLP} / \mathrm{kWh}$ \\
\hline Diesel Start-Up Cost & - & 1000 & $\mathrm{CLP}$ \\
\hline Diesel Price & $C_{D}$ & 500 & $\mathrm{CLP}$ \\
\hline
\end{tabular}

TABLE II: Model parameters

\section{ACKNOWLEDGMENT}

This project is the product of a collaboration between the COMMANDS (INRIA, France) and Centro de Energia teams (Universidad de Chile, Chile).

\section{REFERENCES}

[1] J. Betts, Practical Methods for Optimal Control Using Nonlinear Programming, ser. Advances in design and control. Society for Industrial and Applied Mathematics, 2001.

[2] R. Bellman, Dynamic Programming. Princeton, New Yersey: Princeton Univ. Press, 1957

[3] F. Bonnans, D. Giorgi, S. Maindrault, P. Martinon, and V. Grelard, "Bocop - a collection of examples," INRIA, Tech. Rep., 2014. [Online]. Available: http://www.bocop.org

[4] M. Falcone and R. Ferretti, Semi-Lagrangian Approximation Schemes for Linear and Hamilton-Jacobi Equations. MOS-SIAM Series on Optimization, to appear.

[5] R. Palma-Behnke, C. Benavides, F. Lanas, B. Severino, L. Reyes, and J. Llanos, "A microgrid energy management system based on the rolling horizon strategy," IEEE Transactions on Smartgrids, TSG-00233, vol. R3, 2013 
[6] D. E. Olivares, A. Mehrizi-Sani, A. H. Etemadi, C. A. Cañizares, R. Iravani, M. Kazerani, A. H. Hajimiragha, O. Gomis-Bellmunt, M. Saeedifard, R. Palma-Behnke, G. A. Jiménez-Estévez, and N. D Hatziargyriou, "Trends in microgrid control," IEEE Transactions on Smart Grid, vol. 5, pp. 1905-1919, 2014.

[7] S. Conti, R. Nicolosi, and S. A. Rizzo, "Optimal dispatching of distributed generators in an MV autonomous micro-grid to minimize operating costs and emissions," in IEEE International Symposium on Industrial Electronics, 2010, pp. 2542-2547.

[8] N. Hatziargyriou, G. Contaxis, M. Matos, J. Lopes, G. Kariniotakis, D. Mayer, J. Halliday, G. Dutton, P. Dokopoulos, A. Bakirtzis, J. Stefanakis, A. Gigantidou, P. O’Donnell, D. McCoy, M. Fernandes, J. Cotrim, and A. Figueira, "Energy management and control of island power systems with increased penetration from renewable sources," 2002 IEEE Power Engineering Society Winter Meeting. Conference Proceedings (Cat. No.02CH37309), vol. 1, 2002

[9] C. M. Colson, M. H. Nehrir, and C. Wang, "Ant colony optimization for microgrid multi-objective power management," in 2009 IEEE/PES Power Systems Conference and Exposition, PSCE 2009, 2009.

[10] C. M. Colson, M. H. Nehrir, and S. a. Pourmousavi, "Towards realtime microgrid power management using computational intelligence methods," Power and Energy Society General Meeting 2010 IEEE, pp. $1-8,2007$.

[11] E. Alvarez, A. C. Lopez, J. Gómez-Aleixandre, and N. De Abajo, "On-line minimization of running costs, greenhouse gas emissions and the impact of distributed generation using microgrids on the electrical system," in 1st IEEE-PES/IAS Conference on Sustainable Alternative Energy, SAE 2009 - Proceedings, 2009.

[12] H. Kanchev, D. Lu, B. Francois, and V. Lazarov, "Smart monitoring of a microgrid including gas turbines and a dispatched PV-based active generator for energy management and emissions reduction," in IEEE PES Innovative Smart Grid Technologies Conference Europe, ISGT Europe, 2010.

[13] B. Zhao, X. Zhang, J. Chen, C. Wang, and L. Guo, "Operation optimization of standalone microgrids considering lifetime characteristics of battery energy storage system," IEEE Transactions on Sustainable Energy, vol. 4, pp. 934-943, 2013.

[14] F. Garcia and C. Bordons, "Optimal economic dispatch for renewable energy microgrids with hybrid storage using Model Predictive Control," in IECON 2013 - 39th Annual Conference of the IEEE Industrial Electronics Society, 2013, pp. 7932-7937.

[15] S. A. Pourmousavi, R. K. Sharma, and B. Asghari, "A framework for real-time power management of a grid-tied microgrid to extend battery lifetime and reduce cost of energy," in 2012 IEEE PES Innovative Smart Grid Technologies, ISGT 2012, 2012.

[16] A. Hooshmand, B. Asghari, and R. Sharma, "A novel cost-aware multiobjective energy management method for microgrids," in Innovative Smart Grid Technologies (ISGT), 2013 IEEE PES. IEEE, 2013, pp. $1-6$.

[17] H. J. Khasawneh and M. S. Illindala, "Battery cycle life balancing in a microgrid through flexible distribution of energy and storage resources," Journal of Power Sources, vol. 261, pp. 378-388, 2014.

[18] M. Bardi and I. Capuzzo-Dolcetta, Optimal control and viscosity solutions of Hamilton-Jacobi-Bellman equations, ser. Systems and Control: Foundations and Applications. Birkhäuser, Boston, 1997.

[19] A. Waechter and L. T. Biegler, "On the implementation of an interiorpoint filter line-search algorithm for large-scale nonlinear programming," Mathematical Programming, vol. 106, no. 1, pp. 25-57, 2006.

[20] L. Pontryagin, V. Boltyanski, R. Gamkrelidze, and E. Michtchenko, The Mathematical Theory of Optimal Processes. Wiley Interscience, $\overline{\mathrm{New}}$ York, 1962

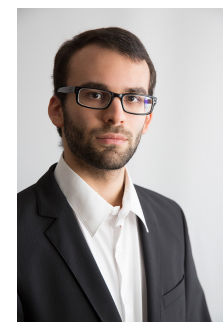

Benjamin Heymann Received a B.Sc. degree in engineering from the Ecole polytechnique (France), a M.Sc. degree in Operations Research from Columbia University in 2012 and 2013 respectively. $\mathrm{He}$ is currently pursuing a Ph.D. in applied mathematics at the Ecole polytechnique. His main research interests are the economics and operations of electricity production.

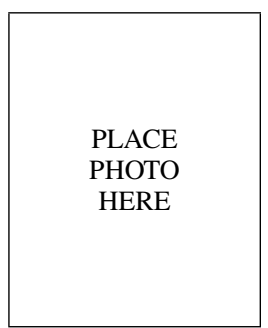

Frederic Bonnans Got an engineering degree from the Ecole Centrale Paris, a PhD (docteur ingénieur) from the Université Technique de Compiègne, and the Habilitation from Université Paris IX Dauphine in 1979, 1982 and 1993 resp. He is currently a senior researcher at Inria Saclay and Ecole polytechnique. His research theme is dynamic optimization for deterministic and stochastic systems, and applications to energy management.

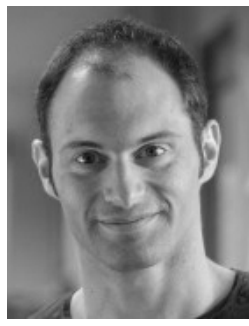

Pierre Martinon Received his M.Sc. and Ph.D degrees in applied mathematics from the ENSEEIHT (Toulouse, France) in 2001 and 2005. He is currently a researcher at Inria Saclay and Ecole polytechnique. His research field is optimization for dynamical systems.

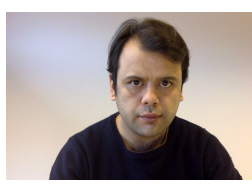

Francisco Silva Received his B.Sc. degree in mathematical engineering from the University of Chile and the $\mathrm{PhD}$ degree in applied mathematics from Ecole polytechnique. Currently, he is an associated professor at the University of Limoges. His main research interests are deterministic and stochastic optimal control theories.

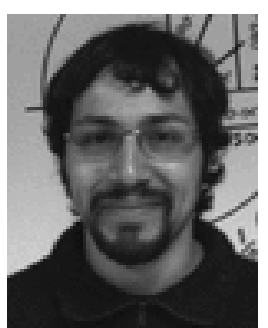

Fernando Lanas Received his B.Sc. degree in electrical engineering from the University of Chile, 2011. His main research interest is the planning and operation of hybrid energy systems based on renewable resources.

PLACE PHOTO

HERE
Guillermo Jiménez Guillermo A. Jiménez-Estvez (IEEE Senior Member) received the B.Sc. degree in electrical engineering from the Escuela Colombiana de Ingenieria, Bogota, in 1998, the M.Sc degree and Ph.D degree in electrical engineering from the Universidad de Chile, Santiago, in 2003 and 2010 respectively. His main research interests are distributed generation and distribution systems planning. 